V.

(A us der Innsbrucker Lnirersitäts-Frauenklinik.)

\title{
Beitrag zur serösen Infiltration des parauterinen Bindegewebes und deren Ausdehnung in weitere Gebiete des Cavum subserosum an der Lebenden. \\ Von
}

\section{E. EHRENDORFER.}

Den Spalträumen des lockeren suoserösen Kellgewebes im Bereiche des Beckens und der ausserhalb desselben sich anschliessenden, weit in die Höhe reichenden retroperitonealen Bindegewebsnassen wurde im Hinblicke auf ihre Bedeutung als vorgebildete Bahnen für die Ausbreitung verschiedener Flüssigkeitsergüsse erst in nenerer 'Leit eine eingehende, wissenschaftliche Würdigung $7 u$ 'Theil. Man versuclite die Maschen und Spalträume des zarten subperitonealen /ellstoffes zumeist auf dem Were künstlicher Infiltrationen (mittelst Iuft und Wasser, Ermeugung künstlicher Oedeme durch Gefässinjection unter starkem Drucke, farbigen l,eiminjectionen unter Frwärmung und constantem Truck), rücksichtlich deren anitomischer Anordnung, sowie Begrenzung einerseits, ihres \%usammenhanges und der Ausdehnungsfähigkeit andererseits, kurz hinsichtlich deren Architektonik kennen $\%$ lernen, um hierdurch über die Mechanik der Ausbreitung der entzündlichen Ergüsse richtige Anschauungen zu erhalten ${ }^{1}$ ).

Eine volle Lebersicht über Sitz und Umfang derartiger Infiltrate, insbesondere des parauterinen Subserosium, sah man nur an einzelnen J seichen oder an Präparaten, deren subperitoneale Räume man nach bekannten Injections- und Sichnittverfahren (Erstarrung der Masse oder Gefrieren lassen) dem näheren Studiun unter\%ogen hatte. Nicht häufig gelang es, gelegrentlich von Eröffnungen der Bauchhöhle, an der laebenden eine klare Einsicht in die pathologisch interessante räumliche Iusclehnung ent\%ündlicher, subserïser Ergüsse \%ugewinnen. Fine seltene (ielegenheit, derartige Verbreitungswege im subperitonealen Bindegewebe an der Lebeuden genau zu übersehen, war mir in cinem Falle ermöglicht, in dem durch eiu chronisches Transudat, das sich ron der 'Tiefe des Beckens bis zur linken Niere hinauf' erstreckte, der Lnterbauchfellraum auf natürlichem Wege in weiter Ausdelınung injicirt war. Die betrefleude Kranke litt an einem

1) Bichat 1802, liönig und Virchow 1S6\%. Henke 157\%, Schlesinger lsi, li. A. F'reund ISSö, Kicgenspeck, Sneguireff-Gubaroff, v. kosthorn, r. Winckl W. A. 
grossen Ltcrusmyom und gew:ihrte in Beckenhochlagerung nach Entfernung der Geschwulst einen vollen Einblick in die gleich $\%$ beschreibenden Veränderungen.

Der liall war folgender: Kr. N. ti Jahre alte Magd aus Fries bei Latndeck, aufgenommen den 24 . 11. 1599. Patientin wurde bald nach Jïntritt ihres Regel im 14. Jahre bleichsüchtig, hat nic zeboren und hatte dic let»te Regel, die, meist schwach, 2-3 Tage ohne Beschwerden zu dauern pflegte, erst vor kur\%en gehalst. Vor etwa $21 / 2$ Jahren litt dic líranke (angeblich nach lirkältung) durch mehrere Wochen in vorühergehenden Schmer\%en und bemerkte damals eine harte Geschwulst im Bauche Da let\%terer allmählich grösser wurle, schickte sie der Ar\%t an die klinik. Appetit, Stuhl und Hiarnentlecrung sollen stets in ()rdnung gewesen sein.

An der mittelgrossen, kräftig gebauten unil mässig genäbrten liranken fand man die Schilddrüse rechts vergrössert, die Jirustorgane gesund. An den Bcinen weder Anschwellungen noch Varices. Der Harn eiweissfrei. Ausser einer gewissen Schwere heim Gehen klagt Pat. weder über Druckerscheinungen noch ausstrahlende Schmerzen in den Beinen. Der Bauch erseheine durch einen harten, über mannskopfgrossen, gut beweglichen 'Tumor (grösster L'mfang $93 \mathrm{~cm}$ ) stark und uugleichmässig vorgewölbt. Der obere Geschwulstpol reicht 2 Querfinger unter den schwertfortsat\%, der unter" beinahe an die Symphyse heran. Die besonders im Querdurehmesser entwickelte ovale Geschwulst reicht bis unter die lippenbögen und \%war mehr nach rechts hin, welcher Antheil dortsell,st auch deutlicher vorspringt. Die Bauchdecken gespannt, über der Geschwulst gedämpfter Schall, Nabelgrube verstrichen. Der innere Genitalbefund ergiebt rirginale Verhialtnisse. Dic Jortio elevirt, steht hinter der Symphyse und bewegt sich bei äusserem Heben der Geschwulst, besonders aber bei Seitenbewegungen derselben deutlich nit. Das hintere Scheidengewölbe erscheint durch die unteren Purthien des derben 'lumors mïssig hervorgewölbt. Der literuskörper ist nicht zu tasten, verliert sich scheinbar ohne schirfe Grenzen in dem Tumor. Die Adnexe liessen sich nicht nachweisen.

Am 1. 12. 1899 laparotomie in Chloroformnarkose und massiger Beckenhochlagerung. [Dor lange, weit über den Nabel hinausreichende Schnitt erüfrete die Bauchdecken, aus deren liindern auffïllig dunkles Blut floss. Die lintwicklung der nirgends angewachsenen Geschwulst gelang mühelos. Es entleerte sich etwa 1/2 Liter leicht blutig gefürbten Ascites. Der Ausgangspunkt der Geschwulst wurcle erst sichtbar. nachdem es gelang, ein anderes, etwa citronengrosses, gestieltes Fibrom, das von links ausging und bei den rechten Adnexen lag (Stieltorsion un et wa 90\%), herauszuheben. Die grosse Geschwulst, die von der hinteren oberen Wand des Fundus uteri ausging, musste um $30-400^{\circ}$ zurückgedreht werden, um den gleichfalls torquirten Cervix gerade \%u stellen. Nach völligem Heratusheben der Geschwulst und Einsetzen grosser Bauchdeckenhacken konate man bei röllig zurūckgesunkenen lecren lïngeweiden sehen, dass das linke ligamentum Iatum weit entfaltet war und zwar durch Insammlung eines serösen Infiltrats, welches von da aus subperitoneal bis in das linke Parancphrium hinaf sich erstreckte.

Das Bauchfell erschien an den erwähnten Stellen glatt glän\%end und \%iemlich gespannt, es \%eigte eive lieihe von buckelartigen lirhühungen mit stellenweisen Vinschnürungen. In der Quere betrug die Ausdehnung des oberen Antheiles etwa Ilandbreite, die Dicke desselben mass etwa 2 J'ingerhōhe. Śehr mïchtig war die susdehnung des fläcbenartig̣ ausgebrciteten Infiltrates, dessen Inhalt $\mathrm{klar}$ erschien und gelblich durchschimmerte, an der Basis des breiten Ligamentes, ron wo sich dasedbe nach abwirts \%u bis gegen das Beckendiaphragma, 7um Theile auch nach rorne gegen das runde Mutterband hin erstreckt. An ein\%elnen Stellen erschien die Anseluwellung im ersten Momente eine durch starke Dubblähun sehr verdünnte Ditrmwind vor\%utinschen. In Folge Eintialtung des Mlesosilpingiums erschien der um die Hälfte vergrüsscrte lierstock ron dem Ende der 'lube weit verschoben und hing auf der durch das seröse Infiltrat stark vorgebuchteten Parthie, mittelst eines etwa kleinfugerdicken Stieles, in den hinteren Douglasraum 
herab. Die linke, stark geschlängelte Tube war verlïngert und stark congestionirt, jedoch nicht entrundlich infiltrirt. Das !inke Ligamentum suspensorium ovarii wurde nach Ahklemmung abgehunden, der linke, anf das Dreilache vergrōsserte lïierstock, welcher ähnlich wie bei Schwangerschaft rin grosses Corpus luteum trug, entfernt. Hierauf wurle das stark geblälte linksseitige Lig:tmentum latum abgebunden und durchtrennt. Beim Durchschneiden entleerte sich eine klare, serise, zum Theile etwas sul\%ige Flüssigkeit.

Das rechte breite Mutterband war mit mächtigen. Venen verselien, nirgends sah man jedoch an dieser Seite eine älnnliche serüse Transsudation, wie im linksseitigen subperitonaalen Binderewebe. Auch hier wurden di. Adnexe nach Abklemmung und doppelter ligatur abgetragen. Schliesslich liess sich, nachdem die runden Mutterbänder durchtrennt waren, auch der $\% u \mathrm{~m}$ fömlichen Tumorsticle ausge\%ogene obere Cervixantheil gut ubersehen. Nach $\Delta$ blösung eines vorderen grösseren, und hinteren kür\%eren Peritoneallappens wurden die seitlichen Uterusgefïsse ligirt, die Arterien isolim versorgt. Niach seitlichem Auset\%en von Kugelzangen wurde das Collum durchtrenot und die Geschwulst entfernt. Keilförmiges Ausschneiden des Cervixstumpfes, Vernähung des Cervicalcanals, hierauf fortlaufende Schlussnaht. Vereinigung der Peritoneallappen nach Einstülpung der ligirten Stümpfe mittelst Leinbert'scher Nilht. Schluss der Ballchdecken in Fitagen. Dic entfernte, etwa $4^{1}$, kg sehwere, ïber mannskopforosse Geschwulst, welohe intramural aus dem hinteren, oberen Kärperantheil sich entwickelt hatte, zeigrte auf dem Durchschnitte, sowie auch auf dem spăteren mikruskopischen Bilde die Beschaffenheit cines derhen fascikulirten fi iblom yomes Im Inneren des Uteruskörpers befinden sich mehrere kleine intrimurale libromknoten. Ausserden vorne links das früher beschriebene. citronengrosse, ehenfalls sehr derbe, jedoch etwils stärker als der grosse fumor durchfenchtete Fibromyom, das an einen daumendicken, fleischigen Stiele hing. Cregen Inde der 2. Woche wurden die Batuchdeckennähte entfern1. Temperatur und Puls waren his dahin völlig normal, ebenso wie das Befinden der Kiranken. Die Bituchwunde per priman geheilt. In Beginn der 3. Woch. nach der Operation trat für 2 Tage hei sonst ungestiortem Wohlbefinden ein massiges lieher anf. Es war geracle die \%eit, wo dic Regel hătte neurrdings eintreten sollen. Der offenbar eingetretene congestive Lustand dürite eine erhöhte Resorption bewirkt haben. Von da an blieb die Temperatur vollstaindig normal, und wurde die Pattientin an 29. 12. bei bestem Wohlswin geheilt entlassen.

In dem eben mitgetheilten Falle sahen wir demnach in überaschender Ausbreitung sowohl das parauterine Subserosium sin. als das Mesenterium der Flexur, weiters unter Abhebung und Vorluchtung des parietalen Blattes das Bauchfell fast der ganzen Darmbeingrube, endlich jenes der linksseitigen liendengegend his in das pararenale Bindegewebe hin durch ein seröses Oedem geschwulstähnlich und breithasig infiltrirt. Ein kleinerer. Theil des Infiltrates erstreckte sich nach vorne gegen don inneren Antheil des Poupart'schen Bandes. In der rorderen Bauchwand war davon ebensowenig etwa; z.u sehen, als wie an der rechten Beckenbauch-Seite überhaupt. Der äberall deutlich fluctuirencle, in Folge unregelmässiger, buckliger Erhabenheiten gelappt aussehende, retroperitoneale lumor war durchscheinenul. stellte ein zusammenlä̈ngendes Ganze dar und endete seitlicl, mit stellenweise überragenden wulstigen Rändern. Von seinem Beckenantheile war der basale der mächtigste, der oberste 'Theil (ler breiten Mutterbänder (Mesosalpingium) erschien wenigm. auseinander gedrängt. Nach abwärts $\%$ erstreckte sich das Infiltrat bis gegen das Beckendiaphragma, nach der medialen 
Sirite und nach hinten $z u$, bis an die Darmkreurbeinfuge hin, dort, wo Einzelne eine bindegewebige Anheftungsstelle, als einc Irt $/$ wischenwand in der Tela subperitonealis, angegeben halien.

Fragen wir uns nach dem A usgangsorte dieses Infiltrates, $\therefore$ geben uns darüber, hinsichtlich dessen Verbreitung, die von verschiedenen Autoren ausgeführten Injectionsmethoden näheren Anfichluss. Es pflegen künstliche Infiltrate, wie man weiss, von rewissen Ausgangspunkten her auch eine bestimmte Richtung rinzuschlagen und sich dann, entsprechend der verschiedenen Anordnung und Anheftung des Peritoneum, weiterzuverbreiten. Ebenso giebt es lekanntlich für entrüncliche Ergüsse typische Verbroitungswege im Gebiete besagter Bindegewelisspaltrïume. An diese Verhältnisse mag, soweit sie bei unserem Fall. interessiren, in Küı\%e erinnert werden.

Injicirt man nämlich ron der (iegend des oberen Antheiles der breiten Mutterbinder aus, so sammelt sich die Flïssigkeit \%unächst in lockeren, perametralen \%ellgewebe an, verdrängt das letztere, entfaltet die breiten Mutterbinder und hebt das Beckenbatuchfell von der Darmbeingrube ab, indem es die crrossen (iefuisss und den Psoas äberdeckt, dehnt weiter links und hinten das Gekröse des s romanum aus und steigt rückwärts sedtlich in retroperitonealen liaume der Wirbelsäule und den spermatical-Gefüssen entlang, indem es gleich\%eitig das wandstindige Bauchfell abheht, bis zur Niere hinaut. Dabei findet die Ausbreitung eines künstlichen Infiltrates nach oben hin nur hei stïrkerem Druck und reichlicherer liüsigkeitsmenge statt. In solchen Fälen lringt aber auch dann die Injektionsmasse weiter \%wischen die Bauchfellfalten des runden Mutterhandes bis \%um Schenkelring, sowie auch in die Tiefo des kleinen Beckens vor. Diese Iushreitung in die liefe der Beckenhiohle findet auch statt, Wenn man seitlich in der (iegend der Basis der Ligamental litta cine Flüssigkeit injicirt, welche sich unter Vorbuchtung der hinturen unteren Parthien des hreiten Bandes und der Douglasfilte dorthin ausbreitet.

Fintsprechend dem Aushreitungswege nun, welchen das Infiltrat in unserem Falle einschlug, lïsst sich daher gemäss den wï̈hnten Injectionsversuchen, sowiv gemäss den dusbreitungen puerperaler Fixsudate und der grossen Ligamenthamatome, auch hieer mit sicherheit annehmen, dass dasselbe \%wischen den liättern des laigamentum latum sinistrum und \%war ron dem oloren und hinteren Antheile soinen Ausgangspunkt nahm, bevor $\therefore$ die schon angegrebene Verbreitungsrichtung eingeschlagen hat. Die Märlitigkeit des Infiltrates, das noben seiner grossen ärtlichen Aushroitung auch las bauchfell ungroühnlich stark abgehoben hatte, roinnert an beschriebene l'raparate, wie sie künstlich nicht rasch, sondern erst nach längerer \%eit durch fortgeset\%te und reichliche Injectionen eryielt worden sind. Das Vorhandensein von \%alilreichen tieferen und seichteren Furchen, welche ein Jiblaltensein derviolen \%arten fïcher- und halkenatigen \%wischen- 
wände und Brücken, die in dic Tiefe zu den Fascien führen, annehmen liessen, deuten gewiss nicht auf ein schnelles Entstehen, sondern auf eine allmählig unter constantem I)rucke entstehende Anfïllung des subserösen Lückensystems hin.

Diese dünneren, blättrigen Verbindungszüge oder dichteren Septa pflegt eine rasch oder unter starkem I)rucke vordringende Plüssigkeit, ebenso wie ler \%unelımende Eiter zumeist bald \%u zorstören, während dieselben hier schön erhalten erschienen.

Wir laben daher hier ein auf natürliche Weise und durch langsam sich vermehrenden Erguss zu Stande crekommenes Infiltrationsresultat betracliten können, welches die Richtigkeit verschiedener künstlicher Injectionen hinsichtlich der Art sowie Richtung der Füllung der lockeren Spalträume im Bindegewebe nur \%u bestätigen vermochte, zumal jener Injectionen, welche unter langanlıaltendeın, gleichmässigem Drucke erzielt worden sind.

Wenn speciell in der Schwangerschaft das subperitoneale Bindegewebe eine rrosse Ausdehnungsfäligkeit und eine gewisse Disposition, grössere Flüssigkeits-Ergüsse aufrunehmen, zu zeigen s(heint, so dürften auch hier bei der allmähligen Entwicklung des grossen Uterusfibroms vielleicht ähnliche Finflïsse wie in jener sich mit geltend gemacht haben. Wie man sonst von maufsteigrenden Abscessen" zu sprechen pflegt, so dïrfte hier von einem naufsteigenden chronischen Oedem " gesprochen werden können. Eine Verwechslung etwa mit anderen Creschwulsthildungen in dieser. Gregend hat hier mit Sicherheit nicht stattmefunden. Intraligamentäre 'Tumoren, als Cysten irgend welcher Art (Parovarial-, serïse Bindegewebs- oder lymphcysten, Lymphangiome, Echinococcus) konnten durch die eigene Art der vorliegenden Aushreitung und die Beschaffenheit der klaffenden stellen bei Iurchtrennung des Iigamentum latum bestimmt ausgeschlossen werden.

Wenn auch die oedematöse S'chwellung nit einem acuten (iufectiösen) Infiltrate eine gewisse Aehnlichkeit hatte, so fanden sich doch, al)gesehen von dem Mangel klinischer Keichen dafür als lieber, Pulserhöhung, Schmerzhattigkeit, ausstrahlende sichuer\%en, Bengestellung des Beines u. s. w., -- weder ein derbes Oedem, trübe Schwellung oder eitrige Infiltration, noch ein injicirtes fibrinös belegtes Bauchfell vor. Ebenso war, schon mit Rücksicht auf den Inhalt, ein Emphysem auszuschliessen.

Was die Ursache dieser chronisch oedematösen Ausschwitzung betrifft, so wird dieselbe wohl mit Bestimmtheit in den mächtigen venösın Plexus des mittleren und tieferen Parametriums (Plexus pampiniformis s. utero-ovarialis) \%u suchen sein. hoi Vorhandensein derartiger grosser Grebirmuttergeschwülste kommt es, ähnlich wie hei Schwangerschaft, zu vielfacher lirweiterung insbesondere der venösen Gefäissolıre und ihrer Aeste, $\% u$ variciosen Ausdehnungen und Verïnderungen ihrer Wand, welche bei gestïrter Blutentleerung \%um Iustritte ron seröser Plüssigkeit in die Cugghung Anlass greben. Auch in unserem 
Falle wird dic Veranlassung $z u$ dem serösen Transsudate darin $\approx \|$ suchen sein, dass in Folge von Zerrung oder Druck, beziehung:weise 'Torsion, ein andauernd behinderter Rückfluss des venösen Blutes und dadurch örtliche Stauung im linksscitigen parametranen Venengrebiete \%ur Bildung des schliesslich sich hoch hinauferstreckenden Oedems geführt hat. Inwieweit sich eine Staumm in den daselbst befindlichen reichlichen Ismphgefüissnet\%en daran betheiligt haben konnte, bleibt dahingestellt.

Es sei noch besonders hervorgehoben, dass der vorhanden. Uterustumor kein fibrocystisches oder in schleimiger Erweichungr begriffenes Fibromyom war. Von einer 'Thrombose umliegendr. Tenen in Parametrium war am Durchschnitte nichts $\% u$ sehen.

Rï̈ckichtlich der 'lorsion des mit den 'Tumor verwachsenen Lteruskörpers wurden zwar Drehungen der Gelärmutter nach künstlichen Injectionen von der Ala respertilionis aus mehrfach beschrieben (die hintere Fläche des Lteruskörpers drehte sich dann einer seitlichen Beckenwand $\% u$ ), trot\%dem erscheint es für. unseren Fall richtiger, auzunehmen, dass diese hier thatsächlich vorhandene Uterusdrehung nicht secundair durch das ausgebreitete natürliche Infiltrat bedingt war, sondern dass die durch das Wachsthum, beziehungsweise durch eine Verschiebung des grossen Fibromyomes primär er\%eugte Uterusdrehung erst secundär die Bildung des 'lranssudates begünstigrt hatte. Eine locale Gefässcirculationsstörung, die in der Hemmung des venösen Abflusses speciell links sich geltend machte, führte schliesslich auch nur zu einer einseitigen Staung und zur Bildung des subserösen Ergusses. Auf G̈rund der erörterten Verhältnisse müssen wir daher künftig darauf Rücksicht nehmen, dass nicht nur entzündliche und Blut-Ergüsse, sondern auch oedematöse Infiltrationen. deren Ausgangsort hauptsächlich im oberen 'Theile des breiten Mutterbandes $z u$ suchen sein wird und die in vereinzelten Fällen bei Gebärmuttergeschwülsten vorkommen können, sich im suhserösen Raume im weitesten Umfange ansbreiten kïnnen.

Obwohl ich gewiss wie auch Andere bei Gelegenheit der Exstirpation von Fibromyomen melufich eine stärkere seröse Durchtränkung des parametralen Zellstoftes angetroffen habe, so habe ich bei Unterleibsgeschwülsten bisher weder einen so hochgradigen, subperitoneal ausgebreitcten serösen Erguss beobachtet, noch konnte ich, soweit ich die Literatur zu äberblicken vermochte, einen ähnlichen Fall beschrieben finden.

In den Capiteln über die Pathologie des Bindegewebe's finden sich, abgesehen von abgesackten 'lumoren, alle möglichen entzündlichen und blutigen Ergüsse im subperitonealen Bindegewebe der breiten Mutterbander und ihrer limgebung unter den Anomalien beschrieben. Von derartigen natürlichen Staunngoedemen findet sich daselbst nichts erwähnt. 